\title{
Effect of single-dose dexmedetomidine on postoperative recovery after ambulatory ureteroscopy and ureteric stenting: a double blind randomized controlled study

\author{
I. I. Shariffuddin ${ }^{1 *}$, W. H. Teoh², S. Wahab ${ }^{1}$ and C. Y. Wang ${ }^{1}$
}

\begin{abstract}
Background: Ambulatory surgery has recently gain popularity, as it is a good method of optimizing hospital resources utilization. To support ambulatory surgery, anaesthetic goals now revolve around patients' early recovery with minimal pain and nausea, expedient discharge home and prompt resumption of activities of daily living. In this study, we evaluated the effect of a single pre-induction dose of dexmedetomidine on anaesthetic requirements, postoperative pain and clinical recovery after ambulatory ureteroscopy andureteric stenting under general anaesthesia.

Methods: Sixty patients were randomised to receive IV dexmedetomidine $0.5 \mu \mathrm{g} . \mathrm{kg}^{-}{ }^{1}$ (Group DEX, $n=30$ ) or IV saline (Group P, $n=30$ ). General anaesthesia was maintained with Sevoflurane: oxygen: air, titrated to BIS 40-60. Pain intensity, sedation, rescue analgesics, nausea/vomiting and resumption of daily activities were recorded at $1 \mathrm{~h}$, and postoperative day (POD) 1-5.
\end{abstract}

Results: Group DEX patients had significant reduction in sevoflurane minimum alveolar concentration (MAC), mean (SD) DEX vs. Placebo 0.6 (0.2) vs. 0.9 (0.1), $p=0.037$; reduced postoperative resting pain at $1 \mathrm{~h}$ (VAS 0-10) (mean (SD) 1.00 (1.84) vs. $2.63(2.78), p=0.004)$, POD 1 (mean (SD) 1.50 (1.48) vs. $2.87(2.72), p=0.002)$, POD 2 (0.53 (0.97) vs. $1.73(1.96), p=0.001)$ and POD 3 (0.30 (0.75) vs. 0.89 (1.49), $p=0.001)$. DEX patients also had less pain on movement POD 1 (3.00 (2.12) vs. 4.30 (3.10), $p=0.043)$ and POD $2(2.10$ (1.98) vs. $3.10(2.46), p=0.040)$, with higher resumption of daily activities by $48 \mathrm{~h}$ compared to placebo, $87 \%$ vs. $63 \%, p=0.04$.

Conclusions: We conclude that a single dose of dexmedetomidine was a useful adjuvant in reducing MAC and postoperative pain (at $1 \mathrm{~h}$ and POD $1-3$ ), facilitating faster return to daily activities by $48 \mathrm{~h}$.

Trial registration: The Australian New Zealand Clinical Trials Registry (ANZCTR), ACTRN12617001120369, 31st July 2017, retrospectively registered.

Keywords: Dexmedetomidine, Ureteroscopy, Minimum alveolar concentration, Pain, Postoperative, Sevoflurane, Recovery

\section{Background}

There has been a paradigm shift in modern healthcare and hospital resource utilization, such that most surgical procedures are now performed in an ambulatory outpatient setting versus traditional hospitalization postoperatively [1]. Anaesthetic goals for ambulatory surgery revolve around patients' early recovery with minimal pain and

\footnotetext{
*Correspondence: inashariffuddin@gmail.com

'Department of Anaesthesia, Faculty of Medicine, University of Malaya, Lembah Pantai, 50603 Kuala Lumpur, Malaysia

Full list of author information is available at the end of the article
}

nausea [2], expedient discharge home and prompt resumption of activities of daily living. However, certain types of operations are associated with higher incidences of severe pain in the early recovery period. After ureteroscopic assisted ureteral stenting, significant flank and bladder pain can occur with reports of pain visual analogue scores (VAS) 6 upon 10 up to 48 h postoperatively [36]. Amelioration of this often necessitates increased parenteral or oral narcotic consumption with attendant side effects of somnolence, postoperative nausea/ vomiting (PONV), clouded sensorium, respiratory 
depression and constipation [7] that are not only distressing but further delay discharge.

Dexmedetomidine is a highly selective $\alpha$-2-agonist exhibiting dose-dependent sedation, analgesia, anti-inflammatory, sympatholytic and anxiolytic effects without relevant respiratory depression $[8,9]$. The intraoperative use of dexmedetomidine has been reported to lower $1 \mathrm{~h}$ postoperative pain scores, reduce opioid consumption and result in a lower risk for opioid-related adverse events by some investigators [10]. However a recent Cochrane review of perioperative dexmedetomidine administration versus placebo in abdominal surgery for adults only managed to show opioid-sparing effects and failed to demonstrate conclusively important differences in postoperative pain scores [11]. The authors concluded that the clinical importance for patients remains uncertain, as the influence of dexmedetomidine on patient-important outcomes such as gastrointestinal function, mobilization and adverse effects could not be satisfactorily determined.

This clinical equipoise left questions unanswered for our institution as to whether it would benefit our patient cohort. We also found no studies evaluating the effect of a single adjuvant dose of dexmedetomidine on patients undergoing ureteroscopy in an ambulatory surgery setting and therefore embarked on this study. We aimed to evaluate the efficacy of a single pre-induction dose of Dexmedetomidine $0.5 \mu \mathrm{g} . \mathrm{kg}^{-}{ }^{1}$ for ureteric stenting after ureteroscopy, and assess its impact on intraoperative anaesthetic agent requirements eg. minimum alveolar concentration (MAC) \{primary outcome\} and secondarily, postoperative pain scores, perioperative analgesia requirements, PONV, drowsiness and return to normal daily activities.

\section{Methods}

The study was approved by our institutional review board (Medical Ethics Committee, University Malaya Medical Centre, ethics no: 672.7) and all patients gave their written informed consent. We enrolled patients of American Society Anaesthesiologists physical status I-II, aged between 18 and 65 years who underwent elective ureteroscopy and ureteric stenting in our ambulatory day care centre. We excluded patients with increased serum creatinine $\left(>200 \mu \mathrm{molL}^{-1}\right)$, advanced liver disease (liver enzymes twice the normal range or higher), those with a history of chronic use of sedatives/ narcotics or analgesics, known alcohol or drug abuse, allergy to study medication and those devoid of postoperative telephone access.

Patients were randomized into two groups, Group Dexmedetomedine (DEX) vs. Placebo using a computer generated random number table in blocks of ten. After recruitment, the enrolling investigators opened sealed opaque envelopes that concealed group allocation.
Participants, trial investigators, attending anaesthetists providing general anaesthesia for the cases, and outcome assesors were blinded to group allocation.

As part of our routine practice, all patients received oral paracetamol $1 \mathrm{~g}$ when they entered the preoperative holding bay. Intravenous access was secured with a 20 Gauge catheter, and ECG, noninvasive arterial blood pressure, heart rate (HR), pulse oximetry and a Bispectral Index (BIS) forehead strip was applied. Haemodynamic variables were recorded at five-minute intervals but BIS monitoring (BIS monitor Model Aspect Medical Systems, Norwood, MA) was only commenced later in the operating room.

In this holding bay, patients randomised to Group DEX then received intravenous dexmedetomedine (Precedex ${ }^{\mathrm{m}}$; Hospira Inc.,Lake Forest,IL,USA) $0.5 \mu \mathrm{g} . \mathrm{kg}^{-1}$ and the placebo group received $20 \mathrm{mls}$ of normal saline. The study drug was prepared by one of the investigators by drawing up the required dose and diluting it with normal saline to a total of $20 \mathrm{ml}$ in a $20 \mathrm{ml}$ syringe, which was then infused over $10 \mathrm{~min}$ via an infusion pump [Perfusor space, B Braun,USA] by a blinded nurse not involved in the trial. Patients in the placebo group received $20 \mathrm{ml}$ normal saline in a similar syringe by the same method. The investigator that prepared the drug had no further role in assessing the patient's outcomes.

Post-administration of the study drug, all patients were transferred to the operating rooms within $10 \mathrm{~min}$, where the patients were positioned on the operating table with their heads resting on a jelly doughnut. Standard monitoring was applied and patients were preoxygenated with $100 \%$ oxygen for $3 \mathrm{~min}$, before anaesthesia was induced with IV fentanyl $1 \mu \mathrm{g} \cdot \mathrm{kg}^{-1}$ and propofol $2 \mathrm{mg} \cdot \mathrm{kg}^{-1}$ or more until patient's exhibited loss of verbal response. A classic laryngeal mask airway size 3 or 4 was then inserted when the jaw was sufficiently slack. Effective ventilation was confirmed on capnography and anaesthesia maintained with Sevoflurane in an oxygen: air mixture. Depth of anaesthesia was titrated to maintain BIS values between 40 to 60 . Intraoperative IV fentanyl in $50 \mu \mathrm{g}$ boluses were given if deemed necessary by the attending anaesthetist managing the case. No routine anti-emetics were administered. The blood pressure, HR, mean alveolar concentration (MAC) of Sevoflurane and BIS values were recorded every $5 \mathrm{~min}$. Upon completion of surgery, the LMA was removed in the operating room when patients were awake and obeying commands.

Postoperatively, patients were assessed in the Post Anaesthesia Care Unit (PACU) within the hour for sedation levels (using Ramsay Sedation Score [12] of $1=$ anxious, agitated or restless; $2=$ cooperative, orientated, tranquil; $3=$ responds to commands; $4=$ asleep but brisk response to light glabellar tap or loud auditory stimulus; $5=$ asleep, 
sluggish response to light glabellar tap or loud auditory stimulus; 6 = asleep, no response), pain intensity (on Visual Analogue Score, VAS 0-10), incidence of nausea and vomiting, need for rescue analgesia (IV fentanyl $25 \mu \mathrm{g}$ boluses) and anti-emetics (IV metoclopramide $10 \mathrm{mg}$ ). Patients were deemed fit for discharge when they were haemodynamically stable, had tolerated a snack (chocolate drink and biscuits) without undue nausea/vomiting, able to stand, void and ambulate with minimal assistance. All patients were discharged home with 5 days worth of oral Celebrex 200 mg b.d.

Two research nurses who were blinded to patient group allocation then conducted a standardised phone interview with the patients daily between 1700 and $1900 \mathrm{~h}$ on postoperative day (POD) $1-5$. Patients were quizzed on their pain at rest (during normal tidal volume breathing) and movement (rising from supine position), incidence of nausea/ vomiting and their ability to ambulate and resume daily activities.

\section{Statistical analysis}

The sample size was based on our primary outcome measure of anaesthetic minimum alveolar concentration (MAC). From an initial pilot study involving 16 patients, we found the mean (SD) MAC of Sevoflurane to be 0.85 (0.19). We deemed a MAC reduction of 0.25 to be a clinically significant difference between the two groups. Prospective power analysis indicated that with $\alpha=0.05$ and power of $80 \%, 25$ patients per group were needed. Therefore we recruited 30 patients per group to account for dropouts. Parametric data and non-parametric data was analysed with Student's t test and Mann-Whitney U-test respectively, and Fisher's exact test was used to compare side effects using SPSS 15.0 ${ }^{\mathrm{Tm}}$ (SPSS Inc., Chicago, IL, USA) software. A $p$ value of $<0.05$ was deemed statistically significant.

\section{Results}

Seventy patients were recruited to the study (Fig. 1) and sixty were eventually randomised after fulfilling eligibility criteria. Baseline demographics of the patients were comparable, barring an older age group in the placebo group occurring by chance (Table 1).

Intraoperatively, we found a significant reduction in the mean (SD) MAC of the anaesthetic agent in patients who had received dexmedetomidine compared to placebo, $0.6(0.2)$ vs. 0.9 (0.1), $p=0.037$ (Fig. 2). There were no differences in haemodynamics between the two groups (recorded from the time the infusion commenced and continued intraoperatively) except at $15 \mathrm{~min}$, where there was a significant lowering of the mean (SD) DEX systolic blood pressure 104.3 (12.8) vs.114.2 (21.2) $\mathrm{mmHg}$, diastolic blood pressure 62.3 (11.8) vs.72.2 (19.2) $\mathrm{mmHg}$, and heart rate 62.6 (10.5) vs. 69.7 (12.1) compared to placebo (all $p<0.05$ ) which then became insignificant at the 20 min mark (Fig. 3).

Intraoperatively both groups received the same amount of fentanyl but the mean (SD) VAS for postoperative pain $1 \mathrm{~h}$ after PACU arrival was significantly reduced in the dexmedetomidine group, 1.00 (1.84) vs. 2.63 (2.78), $p=0.004$. Correspondingly, no patient in the DEX group required rescue fentanyl in the PACU, whereas the placebo group received a median [range] of 0 [0-100] $\mu$, $p=0.040$ (Table 2). There was no difference in the incidence of nausea/ vomiting or use of anti-emetics. Median [range] sedation score for both DEX and placebo were comparable, 2 [2-4] vs. 2 [2-3], $p=0.160$.

Two patients in the placebo group required unexpected hospital admission. One patient had uncontrolled pain postoperatively (VAS 10/10) despite receiving IV Tramadol $100 \mathrm{mg}$ in addition to fentanyl rescue in the PACU and requested admission for pain control and rest for 3 days. Another patient had persistent haematuria and was admitted for observation overnight on a suspicion of sepsis but his haemoglobin level was $12.8 \mathrm{~g} / \mathrm{dL}$, platelets $271 \times 10^{9}$; he remained afebrile with a normal total white count and was discharged the next day.

Upon discharge home, patients that had received DEX had significantly reduced pain scores at rest on the first to third postoperative day, and reduced pain on movement on POD 1 and POD 2 compared to placebo [Table 3]. Significantly more DEX patients resumed their normal daily activities on the 2nd postoperative day than placebo, $26 / 30$ (87\%) vs. $19 / 30$ (63\%), $p=0.037$ [Fig. 4]. On POD 4 patients who received DEX continued to exhibit lower pain scores at rest and upon movement although this did not achieve statistical significance, and by POD5, this advantage was only seen on movement (Table 3 ).

\section{Discussion}

In this study, we demonstrated that a single pre-induction dose of dexmedetomidine $0.5 \mu \mathrm{g} . \mathrm{kg}^{-1}$ administered to patients undergoing ureteroscopy and ureteric stenting, not only significantly reduced the MAC of sevoflurane and intraoperative anaesthetic requirements, but also resulted in a significant $60 \%$ reduction in immediate postoperative pain that extended to Day 3 post discharge. This was highly clinically significant allowing patients earlier resumption of their daily activities and return to the workforce without being hindered by pain occurring after discharge that would negatively impact patient recovery and place economic burdens on patient and caregiver [13].

$\alpha^{2}$-Adrenergic agonists produce clinical effects after binding to $\alpha^{2}$-adrenergic receptors, of which there are three subtypes $\left(\alpha^{2 \mathrm{~A}}, \alpha^{2 \mathrm{~B}}\right.$, and $\left.\alpha^{2 \mathrm{C}}\right)$. These receptor subtypes are distributed ubiquitously, and early mice studies investigating substitution of a mutant $\alpha^{2 \mathrm{~A}}$-adrenergic 


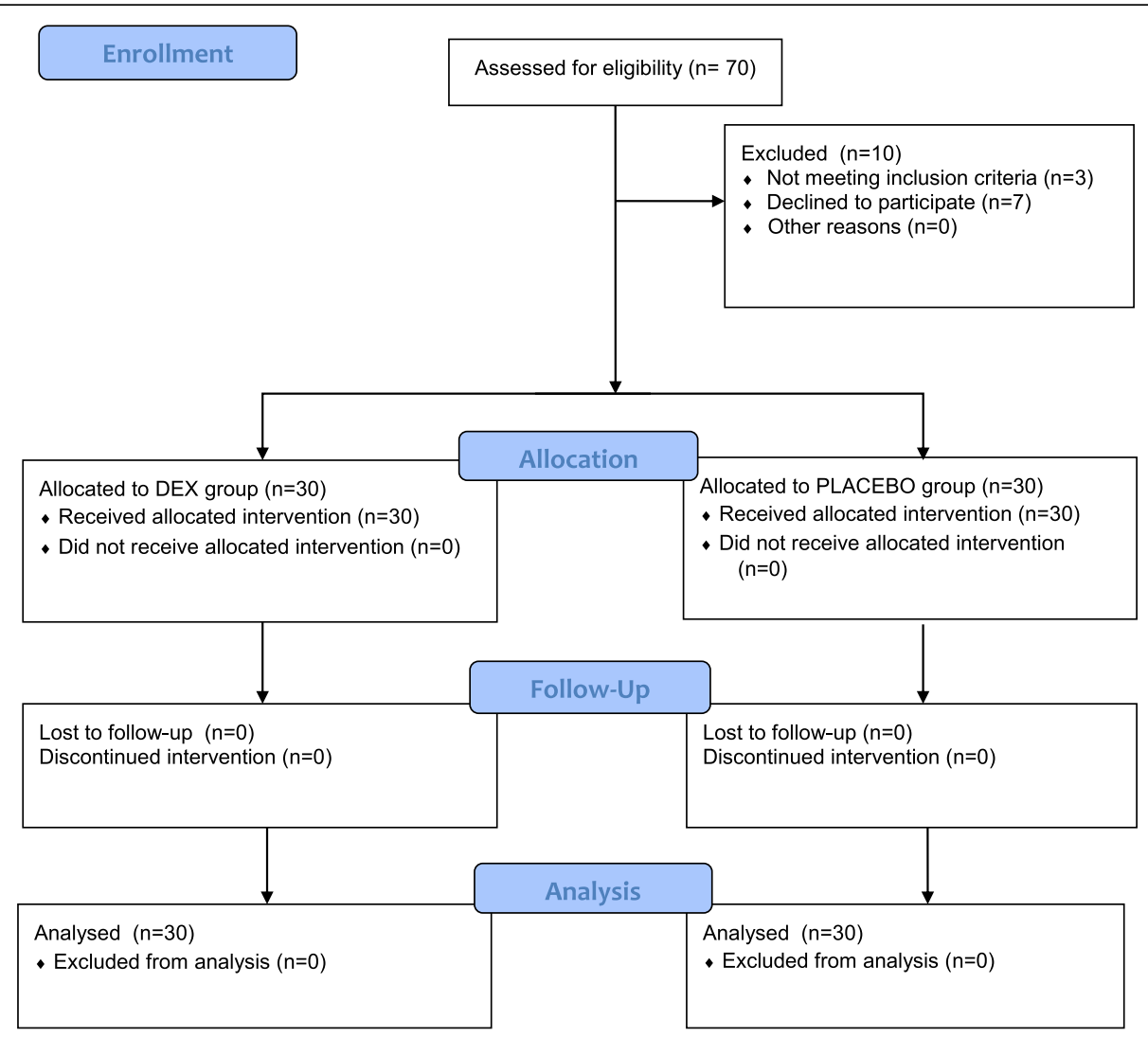

Fig. 1 Flow of patients through the study

receptor via 'hit and run' gene targeting revealed the role of this subtype in sedative, analgesic, and anestheticsparing responses in vivo [14]. In this aspect, our study echoes the findings of other investigations in humans that use minimum alveolar concentration (MAC) as a measure of anesthetic potency. Others have demonstrated that women undergoing abdominal hysterectomy who received dexmedetomidine infusions (target plasma

Table 1 Baseline characteristics of patients receiving intravenous dexmedetomidine $0.5 \mu \mathrm{g} . \mathrm{kg}^{-1}$ or placebo. Values are mean (SD) and numbers of patients (n)

\begin{tabular}{lll}
\hline & $\operatorname{Dex}(n=30)$ & Placebo $(n=30)$ \\
\hline Age; years & $39.2(11.2)$ & $46.3(13.1)$ \\
Weight; $\mathrm{kg}$ & $66.6(9.4)$ & $65.8(13.1)$ \\
ASA class (I: II) & $26 / 4$ & $19 / 11$ \\
Gender (M: F) & $25: 5$ & $22: 8$ \\
Duration of surgery; min & $50.7(22.3)$ & $45.5(15.7)$ \\
Induction dose of propofol; mg & $137.7(22.3)$ & $145.6(35.6)$ \\
Intraoperative fentanyl; $\mu \mathrm{gg}$ & $20.9(21.7)$ & $26.7(18.5)$ \\
Ureteroscopy \& ureteric stenting & & \\
single & 27 & 28 \\
bilateral & 3 & 2 \\
\hline
\end{tabular}

concentration of $0.6 \mathrm{ng} \cdot \mathrm{ml}^{-1}$ ) had a $47 \%$ reduction in the MAC of isoflurane, compared to without dexmedetomidine [15]. Similarly an infusion of dexmedetomidine $0.7 \mathrm{ng} \cdot \mathrm{ml}^{-1}$ administered $15 \mathrm{~min}$ pre- induction of anaesthesia also decreased the MAC of sevoflurane by $17 \%$ in adults aged 55-70 years [16]. Other investigators who used BIS monitoring 40-60 to titrate their end-tidal Sevoflurane concentrations also found a reduction in MAC in surgical patients receiving continuous dexmedetomidine infusions at $0.5 \mu \mathrm{g} . \mathrm{kg}^{\mathrm{h}} \mathrm{hr}^{-1}$ with relative haemodynamic stability [17]. We found this reduction in MAC with just a small single dose of dexmedetomidine administered preoperatively, echoing the findings of Lawrence $\mathrm{CJ}$ et al. who found that a single pre-induction intravenous dose of dexmedetomidine $2 \mu \mathrm{g} \cdot \mathrm{kg}^{-1}$ resulted in lower mean intraoperative isoflurane concentration in the dexmedetomidine-treated patients than controls undergoing minor orthopaedic and general surgery [18].

The analgesic properties of dexmedetomidine are conferred by its central action at the locus coeruleus in the brainstem and spinal cord that inhibits neuronal firing, thereby triggering sedation, anxiolysis and analgesia [19]. We found that a single pre-induction dose of dexmedetomidine $0.5 \mu \mathrm{g} . \mathrm{kg}^{-1}$ reduced immediate postoperative pain by a dramatic $60 \%$ in our study. This resonates with 


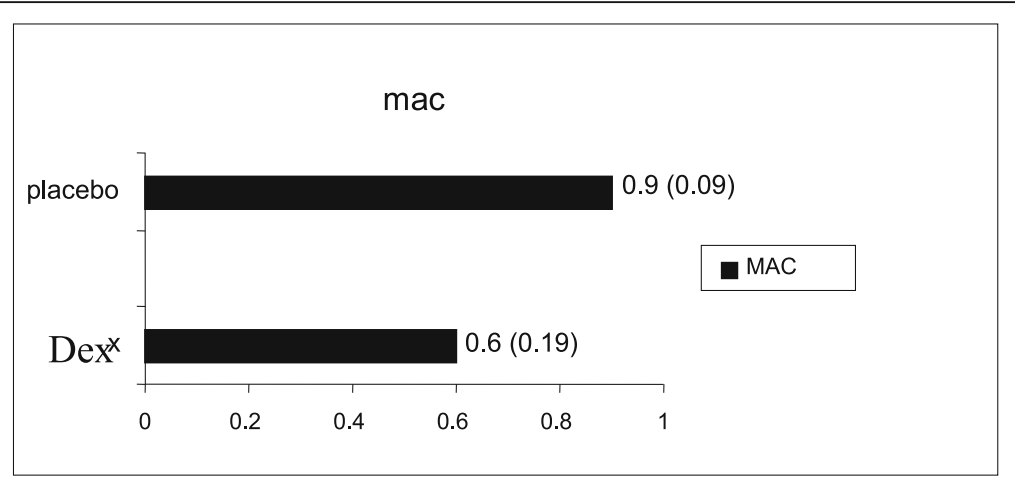

Fig. 2 Mean (SD) minimum alveolar concentration (MAC) of patients who received dexmedetomidine compared to placebo.

Schnabel's [10] meta-analysis of 28 randomised controlled trials involving 1420 adults that also reported lower postoperative pain at $1 \mathrm{~h}$ (but unlike them we did not find any opiod sparing effects or significant intraoperative bradycardia). Further analyzing 11 randomized controlled trials,
434 children receiving dexmedetomidine demonstrated a reduced relative risk for postoperative pain in comparison with placebo, although the influence of dexmedetomidine on postoperative opioid consumption was less clear in this meta-analysis [20].

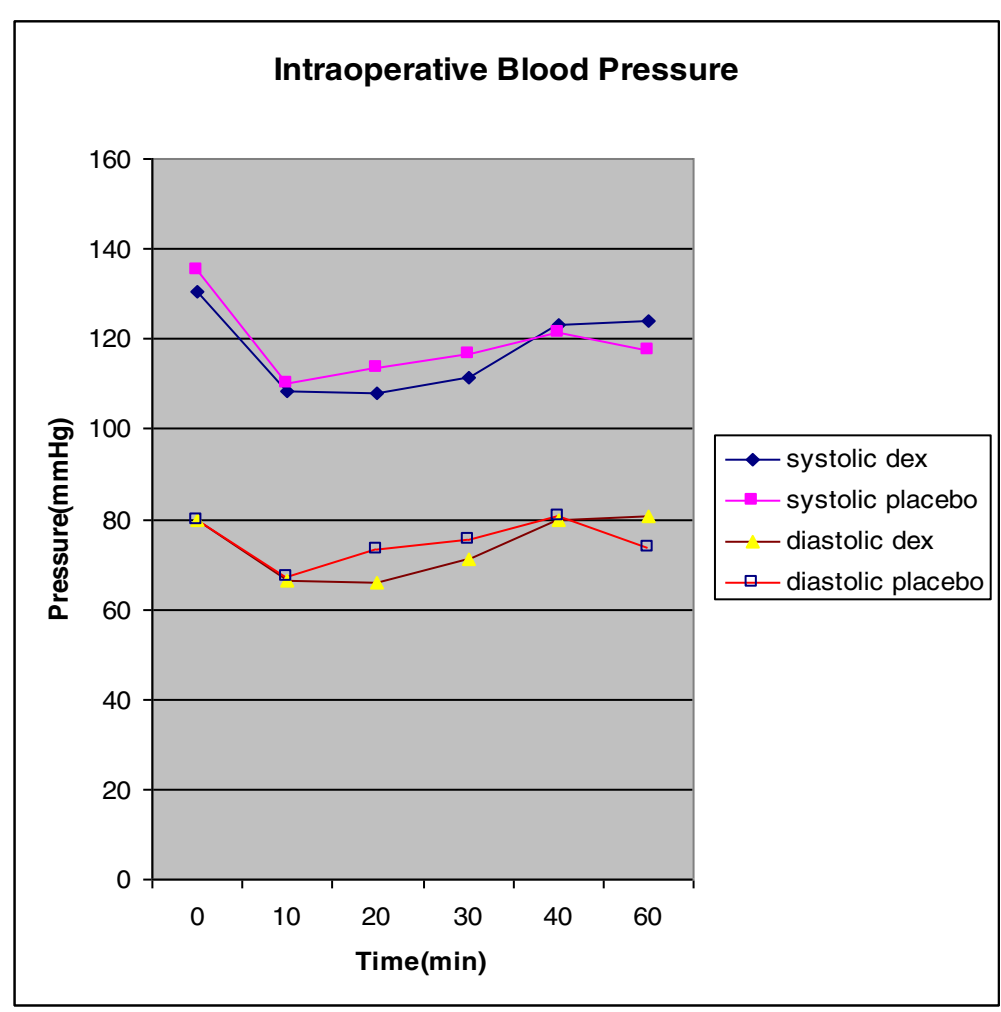

Legends:
$\longrightarrow$ Systolic dex:
Systolic Blood pressure of Dexmedetomidine
-
Systolic Blood pressure of Placebo
- Diastolic dex:
Diastolic blood pressure of Dexmedetomidine
$\square$ Diastolic placebo:
Diastolic Blood pressure of Placebo

Fig. 3 Intraoperative haemodynamic changes 
Table 2 Post-anaesthesia care unit (PACU) recovery profile of patients who received intravenous dexmedetomidine $0.5 \mu \mathrm{g} . \mathrm{kg}^{-1}$ or placebo. Values are mean (SD), median [range], numbers of patients (n) or proportions (\%)

\begin{tabular}{llll}
\hline & Dex $(n=30)$ & Placebo $(n=30)$ & $P$ Value \\
\hline PACU fentanyl; $\mu \mathrm{g}$ & 0 & $0[0-100]$ & $0.040^{*}$ \\
PACU anti-emetics; Yes / No & $0: 30$ & $4: 26$ & 0.110 \\
Ramsay Sedation Score & $2[2-4]$ & $2[2-3]$ & 0.160 \\
Nausea and vomiting & $0 / 30(0 \%)$ & $2 / 30(6.7 \%)$ & 0.492 \\
Unexpected admission & $0 / 30(0 \%)$ & $2 / 30(6.7 \%)$ & 0.492 \\
\hline
\end{tabular}

*Indicates statistically significant $p$ value

On the other hand, other investigators have found no difference in immediate postoperative pain but a reduced amount of rescue analgesics after a single dose of dexmedetomidine [21]. A recent Cochrane review [11] that included 402 adults undergoing abdominal surgery who received dexmedetomidine found a reduction in 'rescue' opioid consumption, but no clinically perceptible differences in postoperative pain (visual analogue scale (VAS) 0 to $100 \mathrm{~mm}$, where $0=$ no pain and $100=$ worst imaginable pain) in the first $24 \mathrm{~h}$ after surgery when compared with placebo. However the authors opined that the quality of the evidence was very low as the result of imprecision, methodological limitations and substantial heterogeneity among the six included studies.

We demonstrated that a single pre-induction dose of dexmedetomidine $0.5 \mu \mathrm{g} \cdot \mathrm{kg}^{-1}$ resulted in significantly

Table 3 Pain scores at rest and upon movement in the Post-Anaesthesia Care Unit (PACU) and on postoperative day (POD) 1-5. Values are mean (SD)

\begin{tabular}{llll}
\hline Drugs & Dex $(n=30)$ & Placebo $(n=30)$ & $P$ Value \\
\hline PACU & $1.00(1.84)$ & $2.63(2.78)$ & $0.004^{*}$ \\
POD1 & & & \\
Rest & $1.50(1.48)$ & $2.87(2.72)$ & $0.002^{*}$ \\
Movement & $3.00(2.12)$ & $4.30(3.10)$ & $0.043^{*}$ \\
POD2 & & & \\
Rest & $0.53(0.97)$ & $1.73(1.96)$ & $0.001^{*}$ \\
Movement & $2.10(1.98)$ & $3.10(2.46)$ & $0.040^{*}$ \\
POD3 & & & \\
Rest & $0.30(0.75)$ & $0.89(1.49)$ & $0.001^{*}$ \\
Movement & $1.60(1.73)$ & $2.10(1.98)$ & 0.630 \\
POD4 & & & 0.276 \\
Rest & $0.17(0.64)$ & $0.29(0.71)$ & 0.987 \\
Movement & $0.80(1.56)$ & $1.00(1.49)$ & 0.923 \\
POD5 & & & 0.308 \\
Rest & $0.07(0.36)$ & $0.07(0.37)$ & \\
Movement & $0.30(1.95)$ & $0.77(1.40)$ & \\
\hline
\end{tabular}

*Indicates statistically significant $p$ value reduced pain at rest for the first three postoperative days, and pain on movement on postoperative day 1 and 2. More importantly, our study significantly showed that this extended analgesic duration actually enhanced the recovery profile and enabled a significantly higher proportion of patients to return to their daily activities after $48 \mathrm{~h}$ as compared to the placebo group. In a rat model of incisional pain, pretreatment with dexmedetomidine (administered subcutaneously 30 min before plantar incision) significantly decreased remifentanil-induced postoperative hyperalgesia as indicated by increased paw withdrawal latencies and thresholds to thermal and mechanical stimulation [22]. Western blotting experiments revealed that the antihyperalgesic effects of dexmedetomidine were associated with suppression of spinal cord N-methyl-d-aspartate receptor (NMDAR) excitability, as measured by a reduction in NR2B (NMDR receptor 2B subunit) tyrosine phosphorylation (Tyr1472 site), which was upregulated after remifentanil infusion [22]. These results correlated with the rat's pain behavior changes suggesting that dexmedetomidine could efficiently alleviate opiod-induced hyperalgesia and we postulate this to be the underlying reason for its extended analgesic benefits beyond its half-life, even though administered as a single dose.

Furthermore, surgical injury to tissue results in increased stress hormone production (C-reactive protein, cortisol, catecholamines), complement system activation, leukocyte migration to the site of injury, and release of cytokines (interleukins, tumor necrosis factor), superoxide radicals, proteases and growth factors [23]. Although an appropriate inflammatory cascade is essential for tissue reconstitution, restoration of homeostasis and infection control, these inflammatory mediators may induce fatigue and prolong convalescence in otherwise healthy patients. The choice of anaesthetic technique can affect both immunostimulatory and immunosuppressive mechanisms; directly by modulating immune cell function or indirectly by attenuating the stress response thereby impacting short and long-term patient outcomes.

There is already evidence that dexmedetomidine decreases production of inflammatory cytokines while lowering intra-abdominal pressure in critically ill patients with sepsis [9]. In animals, dexmedetomidine attenuates the increase of plasma cytokine levels after endotoxin injection and drastically reduces the mortality rate of infected animals [24]. These results lend weight to the role of dexmedetomidine in preventing untoward stress responses, possibly mitigating delays in post-surgical convalescence and enhancing quality of recovery from surgery. Indeed, Bekker et al. found that patients who were administered a dexmedetomidine infusion during multilevel spinal fusions exhibited an improved quality of recovery (global 40-item quality of recovery questionnaire scores, QoR40) and reduced fatigue (assessed using Fatigue 


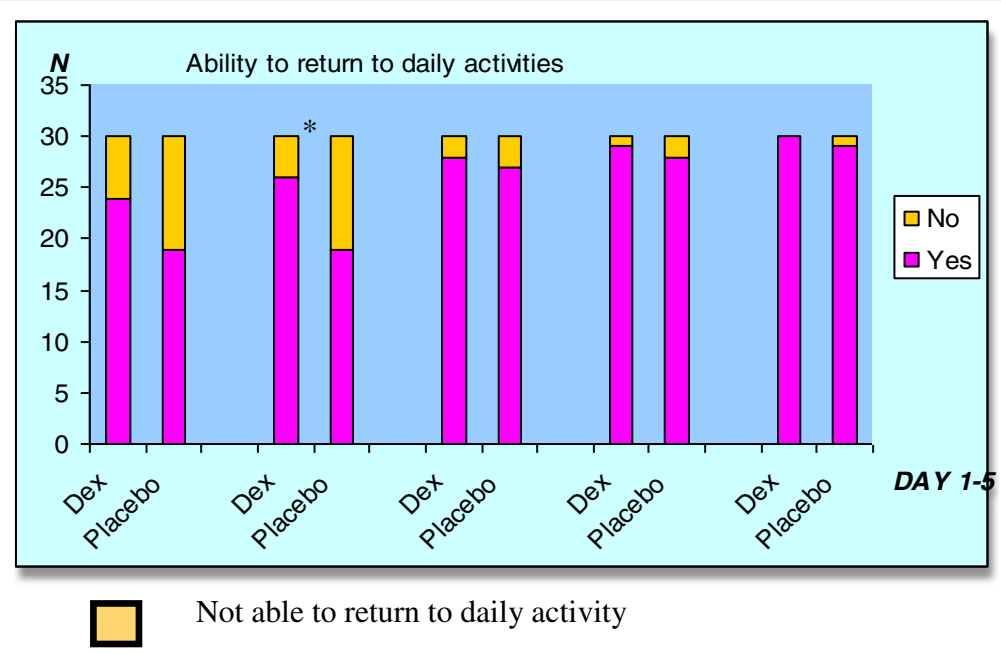

Able to return to daily activity

$\mathrm{P}$ value $<0.037$

Fig. 4 Number of patients who were able to resume their daily activities on postoperative Day 1-5

Severity Scores, FSS) on the third postoperative day, with reduced plasma cortisol and interleukin10 levels in comparison with the control group [25]. Using the same QoR40 and FSS benchmarks, Ge et al. also found that abdominal hysterectomy patients who received a propofol/ remifentanil/ dexmedetomidine anaesthetic showed higher recovery scores from the third postoperative day [26]. These data support our own study's and explains how a single dose of dexmedetomidine could confer far-reaching extended benefits to our patient cohort in terms of reduced postoperative pain for 3 days and faster return to their regular lifestyles.

Our study did have the following limitations: preoperative assessment could have ideally screened for motion sickness and smoking as possible confounders of the risk of PONV. We did not measure plasma catecholamine or stresshormone concentrations; although these measurements could support a direct relationship between sympatholytic and anti-inflammatory properties of dexmedetomidine and postoperative quality of recovery, it was not logistically feasible under the constraints of our study setting. Additionally as MAC decreases $4 \%$ to $5 \%$ per degree centigrade decrease in core temperature [15], temperature monitoring could have been applied. However we did not do this as surgery was relatively short averaging 45-60 $\mathrm{min}$, and we had applied a forced air warmer to the upper bodies of all patients to maintain normothermia.

\section{Conclusions}

In conclusion, we found that the administration of a single pre-induction dose of dexmedetomidine $0.5 \mu \mathrm{g} \cdot \mathrm{kg}^{-1}$ reduced intraoperative anaesthetic agent requirements, alleviated immediate postoperative pain in hospital and up to the third postoperative day at home, enabling patients earlier resumption of their daily activities. We conclude that dexmedetomidine proved to be a useful adjuvant that benefitted our patients undergoing ureteroscopy and ureteric stenting in our ambulatory clinical practice.

\section{Abbreviations}

BIS: Bispectral Index; DEX: Dexmedetomedine; FSS: Fatigue Severity Scores; HR: Heart rate; MAC: Minimum alveolar concentration; PONV: Postoperative nausea/vomiting; QoR40: global 40-item quality of recovery questionnaire scores; VAS: Visual analogue scores

\section{Acknowledgements}

None

\section{Funding}

This clinical trial received research funding from University of Malaya, Shortterm research fund (Grant no. PJP FS345/2008C). Funding does not play any role in the design of study, collection, analysis and interpretation of data and in writing the manuscript.

\section{Availability of data and materials}

All datasets on which the conclusions of the manuscript are presented in the main paper and the data are kept in Dept of Anaesthesia, University Malaya. Contact person is Madam Sujithara Devi A/P Arumugam, Email: suji86@um.edu.my

\section{Authors' contributions}

IIS: study design, data collection, data analysis, patient recruitment, revising the article critically for important intellectual content and final approval of the version to be submitted. WHT: data analysis, revising the article critically for important intellectual content and final approval of the version to be submitted. SW: data collection, patient recruitment and final approval of the version to be submitted. CYW: study design, data analysis and final approval of the version to be submitted. All authors read and approved the final manuscript. 


\section{Ethics approval and consent to participate}

The study approved by University Malaya Medical Centre Institutional Review Board Reference 672.7 .

All patients signed a written informed consent before enrolment.

\section{Consent for publication}

Consented by participant.

\section{Competing interests}

The authors declare that they have no competing interests.

\section{Publisher's Note}

Springer Nature remains neutral with regard to jurisdictional claims in published maps and institutional affiliations.

\section{Author details}

'Department of Anaesthesia, Faculty of Medicine, University of Malaya, Lembah Pantai, 50603 Kuala Lumpur, Malaysia. ${ }^{2}$ Wendy Teoh Pte. Ltd, Private Anaesthesia Practice, Singapore, Singapore.

Received: 14 August 2017 Accepted: 18 December 2017

\section{Published online: 05 January 2018}

\section{References}

1. Cullen KA, Hall MJ, Golosinskiy A. Ambulatory surgery in the United States, 2006. Natl Health Stat Report. 2009:11:1-25.

2. Van VLymen JM, White PF. Fast track concept for ambulatory anaesthesia. Curr Opin Anaesthesiol. 1998;11(6):607-13.

3. Stoller ML, Wolf JS Jr, Hoffman R, Marc B. Ureteroscopy without routine balloon dilatation: an outcome assessment. J Urol. 1992;147(5):1238-42.

4. Hosking DH, McColm SE, Smith WE. Is stenting following ureteroscopy for removal of distal ureteral calculi necessary? J Urol. 1999;161(1):48-50.

5. Cheung MC, Yip SK, Lee FC, Tam PC. Outpatient ureteroscopy lithotripsy: selective internal stenting and factors enhancing success. J Endourol. 2000 14(7):559-64.

6. Borboroglu PG, Amling CL, Schenkman NS, Monga M, Ward JF, Piper NY, Bishoff JT, Kane CJ. Ureteral stenting after ureteroscopy for distal ureteral calculi: a multi institutional and prospective randomized controlled study assessing pain, outcomes and complications. J Urol. 2001;166(5):1651-7.

7. Sinclair DR, Chung F, Mezei G. Can postoperative nausea and vomiting be predicted? Anesthesiology. 1999;91:109-18.

8. Paris A, Tonner PH. Dexmedetomidine in anaesthesia. Curr Opin Anaesthesiol. 2005;18:412-8.

9. Tasdogan M, Memis D, Sut N, Yuksel M. Results of a pilot study on the effects of propofol and dexmedetomidine on inflammatory responses and intraabdominal pressure in severe sepsis. J Clin Anesth. 2009:21:394-400.

10. Schnabel A, Meyer-Friessem CH, Reichl SU, Zahn PK, Pogatzki-Zahn EM. Is intraoperative dexmedetomidine a new option for postoperative pain treatment? A meta-analysis of randomized controlled trials. Pain. 2013;154:1140-9.

11. Jessen Lundorf L, Korvenius Nedergaard H, Møller AM. Perioperative dexmedetomidine for acute pain after abdominal surgery in adults. Cochrane Database Syst Rev. 2016;2:CD010358.

12. Ramsay MA, Savege TM, Simpson BR, Goodwin R. Controlled sedation with alphaxalone-alphadolone. Br Med J. 1974;2(5920):656-9.

13. Wu CL, Berenholtz SM, Pronovost PJ, Fleisher LA. Systematic review and analysis of postdischarge symptoms after outpatient surgery. Anaesthesiology. 2002;96:994-1003.

14. Lakhlani PP, MacMillan LB, Guo TZ, McCool BA, Lovinger DM, Maze M, Limbird LE. Substitution of a mutant a2A-adrenergic receptor via 'hit and run' gene targeting reveals the role of this subtype in sedative, analgesic, and anesthetic-sparing responses in vivo. Proc Natl Acad Sci U S A. 1997:94:9950-5.

15. Aantaa R, Jaakola ML, Kallio A, Kanto J. Reduction of the minimum alveolar concentration of isoflurane by dexmedetomidine. Anesthesiology. 1997;86: 1055-60.

16. Fragen RJ, Fitzgerald PC. Effect of dexmedetomidine on the minimum alveolar concentration (MAC) of sevoflurane in adults age 55 to 70 years. J Clin Anesth. 1999;11:466-70.

17. Magalhães E, Govêia CS, Ladeira LC. Espíndola BV.[relationship between dexmedetomidine continuous infusion and end-tidal sevoflurane concentration, monitored by bispectral analysis.]. Rev Bras Anestesiol. 2004; 54(3):303-10.
18. Lawrence CJ, De Lange S. Effects of a single pre-operative dexmedetomidine dose on isoflurane requirements and perioperative hemodynamic stability. Anesthesia. 1997;52:736-44.

19. Kamibayashi T, Maze M. Clinical uses of alpha2 -adrenergic agonists. Anesthesiology. 2000;93(5):1345-9.

20. Schnabel A, Reichl SU, Poepping DM, Kranke P, Pogatzki-Zahn EM, Zahn PK. Efficacy and safety of intraoperative dexmedetomidine for acute postoperative pain in children: a meta-analysis of randomized controlled trials. Paediatr Anaesth. 2013:23:170-9.

21. Kim SH, Oh YJ, Park BW, Sim J, Choi YS. Effects of single-dose dexmedetomidine on the quality of recovery after modified radical mastectomy: a randomised controlled trial. Minerva Anestesiol. 2013;79(11): 1248-58.

22. Zheng Y, Cui S, Liu Y, Zhang J, Zhang W, Gu X, Ma Z. Dexmedetomidine prevents remifentanil-induced postoperative hyperalgesia and decreases spinal tyrosine phosphorylation of $\mathrm{N}$-methyl-d-aspartate receptor $2 \mathrm{~B}$ subunit. Brain Res Bull. 2012;87:427-31.

23. Kurosawa $\mathrm{S}$, Kato M. Anesthetics, immune cells, and immune responses. J Anesth. 2008;22(3):263-77.

24. Taniguchi T, Kidani Y, Kanakura H, Takemoto Y, Yamamoto K. Effects of dexmedetomidine on mortality rate and inflammatory responses to endotoxin-induced shock in rats. Crit Care Med. 2004;32(6):1322-6.

25. Bekker A, Haile M, Kline R, Didehvar S, Babu R, Martiniuk F, Urban M. The effect of intraoperative infusion of dexmedetomidine on the quality of recovery after major spinal surgery. J Neurosurg Anesthesiol. 2013;25(1):16-24.

26. Ge DJ, Qi B, Tang G, Li JY. Intraoperative Dexmedetomidine promotes postoperative analgesia and recovery in patients after abdominal hysterectomy: a double-blind, randomized clinical trial. Sci Rep. 2016;6: 21514. https://doi.org/10.1038/srep21514.

\section{Submit your next manuscript to BioMed Central and we will help you at every step:}

- We accept pre-submission inquiries

- Our selector tool helps you to find the most relevant journal

- We provide round the clock customer support

- Convenient online submission

- Thorough peer review

- Inclusion in PubMed and all major indexing services

- Maximum visibility for your research

Submit your manuscript at www.biomedcentral.com/submit
) BioMed Central 